\title{
Multiple Odontogenic Keratocysts in Nonsyndromic Patients - A Case Report and Review of Literature
}

\author{
Shivani K Jamwal ${ }^{1}$, Kirthi K Rai ${ }^{2}$, Chatura K Ramakanth, Umashankara K Veerbhadrappa, Nelagondanahalli T Geetha ${ }^{5}$
}

\begin{abstract}
Aim: The aim of this study is to highlight a behavioral pattern of odontogenic keratocysts (OKCs) in syndromic and nonsyndromic patients in terms of clinical course, genetic makeup, local destruction, management modalities, and recurrences such that detection in initial stages and intervention when done prevents extensive morbidity and a better quality of life.

Background: OKC is a common developmental odontogenic cyst affecting the oral and the maxillofacial region, which arises from the dental lamina or its remnants. Multiple OKCs are usually seen in association with the nevoid basal cell carcinoma syndrome (NBCCS) but approximately $5 \%$ of the cases have multiple cysts without concomitant syndromic presentation. As it spreads by way of the bone marrow, it results in more destruction before any clinical manifestation appears. Although lesions are benign but are aggressive, locally destructive, and highly recurrent. Case description: A 15-year-old male patient, a rare case of nonsyndromic multiple OKCs, is presented here along with a review of literature with emphasis on the importance of thorough evaluation, diagnosis, treatment strategy, and strict follow-up.

Conclusion: Although our case does not fulfill the major and minor criteria of syndrome, but considering the reported literature and clinical presentation, early age, multiple OKCs, involvement of both the jaws, cleft lip and cleft palate, and histological correlation, we suggest our case to be a partial expression of NBCCS.

Clinical significance: Any case presenting with single or multiple OKCs should be thoroughly investigated locally and systemically for any other signs of NBCCS or other syndrome. A definite treatment protocol should be formulated depending on patient's age, number and size of lesion, remaining subsequent growth, and proximity to adjacent vital structures, to provide a better quality of life with a minimal amount of morbidity and kept on strict regular follow-up.
\end{abstract}

Keywords: Mandible, Maxilla, Multiple odontogenic keratocysts, NBCCS (Gorlin-Goltz syndrome), OKC.

Journal of Contemporary Dentistry (2019): 10.5005/jp-journals-10031-1252

\section{BACKGROUND}

OKC is a developmental cyst of the odontogenic origin that accounts for $10-15 \%$ of all of the jaw cysts and is characterized by aggressive clinical behavior and high recurrence rate. It arises from cell rests of the dental lamina (Neville et al., 1995). In 2005, World Health Organization (WHO) renamed OKC as keratocystic odontogenic tumor (KCOT) based on its aggressiveness, infiltrative nature, histology, and genetic behaviour." WHO defined KCOT as "a benign uni- or multicystic, intraosseous tumor of odontogenic origin, with a characteristic lining of parakeratinized stratified squamous epithelium with a potential for aggressive, infiltrative behavior". ${ }^{2}$ The 2005 WHO "recommended the term keratocystic odontogenic tumor as it better reflects its neoplastic nature of the lesion." Term OKC was given by Philipsen in 1960 and described by Pindborg and Hansen in 1963. Its clinical and histological features were confirmed by Browne in 1970 and 1971.

OKCs can occur as sporadic or multiple forms, both when associated with syndromes as well as nonsyndromic cases. Multiple OKCs have been found to be commonly associated with NBCCS or the GorlinGoltz syndrome but also have been reported to be associated with the Orofacial Digital Syndrome, Ehler-Danlos syndrome, Noonan syndrome, and Simpson-Golabi-Behmel syndrome. OKCs when associated with NBCCS occur earlier in life and are associated with higher tendency to recur and are more aggressive than nonsyndromic cases and occasionally may get transformed into aggressive neoplasms such as ameloblastoma and squamous cell carcinoma. ${ }^{4}$

OKCs are the most common form of cystic lesions affecting the maxillofacial region with an incidence rate of $12-14 \%$ and two peaks around the ages of 30 and 60 years, and seem to be more frequent
1,4,5 Department of Oral and Maxillofacial Surgery, Bapuji Dental
College and Hospital Davangere, Davangere, Karnataka, India
${ }^{2}$ Department of Oral Maxillofacial and Reconstructive Surgery, Bapuji
Dental College and Hospital Davangere, Davangere, Karnataka, India
${ }^{3}$ Department of Pathology, JJM Medical College, Davangere, Karnataka, India

Corresponding Author: Shivani K Jamwal, Department of Oral and Maxillofacial Surgery, Bapuji Dental College and Hospital Davangere, Davangere, Karnataka, India, Phone: +919076801606, e-mail:dr.sj666@gmail.com

How to cite this article: Jamwal SK, Rai KK, et al. Multiple Odontogenic Keratocysts in Nonsyndromic Patients-A Case Report and Review of Literature. J Contemp Dent 2019;9(1):46-52.

Source of support: Nil

Conflict of interest: None

in males (M/F 2:1). Around $60-80 \%$ of the reported cases occur in the mandible, mainly in the molar, angle, and ramus areas. Usually, a localized asymptomatic swelling is the most common symptom; spontaneous drainage of the cyst into the oral cavity and teeth mobility are also common. Radiographic appearances of OKCs are a unilocular or a multilocular radiolucency with scalloped and well-defined margins, mainly located in the aforementioned area of the mandible. ${ }^{5}$

NBCCS is associated with a triad of multiple basal nevi, multiple OKCs, and skeletal abnormalities. These multiple lesions may be the first manifestation of the NBCCS or otherwise, it may be attributed to the multifocal nature of OKCs. ${ }^{4}$ This syndrome is known to express a high degree of penetrance, variable expressivity, and is 
characterized by several developmental defects. Among patients with NBCCS, $65-75 \%$ have OKCs. These may be bilateral, may involve both jaws, and most commonly arise in the lower third molar and maxillary canine regions. OKCs associated with NBCCS occur at least a decade earlier than cases of isolated OKCs. ${ }^{5}$

Here, we present a rare case report of multiple OKCs with multiple impacted teeth and operated the cleft lip and palate in a 15-year-old male patient, which may be suggestive of partial expression of the PTCH gene.

\section{Case Description}

A 15-year-old male patient reported at the Department Oral and Maxillofacial Surgery, Bapuji Dental College and Hospital Davangere, with the chief complaint of swelling in the left lower one-third of face since two and half months that was not associated with any pain or any fluid discharge. There was no history of trauma in the past. The patient gives a history of cleft lip surgery at the age of 3 months and cleft palate surgery at the age of 9 months. There was no relevant past dental and family history and all vital signs were within normal limits.

Extraoral examination revealed a diffuse swelling over the left lower one-third of face approximately $5 \times 3 \mathrm{~cm}$ in size with overlying normal skin color extending anterio-posteriorly from the left corner of the mouth to $2 \mathrm{~cm}$ anterior to the tragus of the ear on the left side and superior inferiorly from the ala tragus line to the inferior border of the mandible. There was no evidence of any visible pulsations or a draining sinus. On palpation, it was soft to firm in consistency with the overlying skin being pinchable, non-tender, and with no rise in local temperature of the overlying skin (Fig. 1).

Intraoral examination revealed multiple retained deciduous teeth $52,53,55,63,74,75$, and 85 . A diffuse swelling was evident over the left side of the mandibular in relation to 32,33 , and 74 . It was non-tender, fluctuant, soft to firm in consistency with no draining sinus, and it was obliterating the buccal vestibule. Another asymptomatic swelling measuring $2 \times 2 \mathrm{~cm}$ was evident in relation to 75,36 , and 37 regions and it was associated with buccal vestibule obliteration. Also, vestibular obliteration was evident in relation to 24,25 , and 26 regions without an obvious swelling. There was also an evidence of reverse overjet and high arched palate (Fig. 2). In a panoramic radiograph, four radiolucencies with corticated border were revealed around unerupted teeth in relation to first, second, and third quadrants and the left ramus region in relation to 14 and 15 regions on the right side and in relation to 25,26 , and 27 regions on the left side and in the left side of the mandible in relation to 31 , $32,33,34$, and 35 regions and distal to the 37 th region extending toward ramus of the mandible, suggestive of multiple cysts in the first, second, and third quadrants (Fig. 3).

The radiographs from the chest and the skull were unremarkable and no cutaneous abnormality was evident. The CBCT scans revealed multiple hypodense areas involving both maxilla and mandible, lesions were situated in the posterior aspect of both the jaws, a lesion on the right side of the maxilla was bilocular, and there was evidence of the cleft palate in the incisor region. The expansion of the cortical bones of the inferior and posterior borders on the left side of the mandible and bilateral aspects of the maxilla
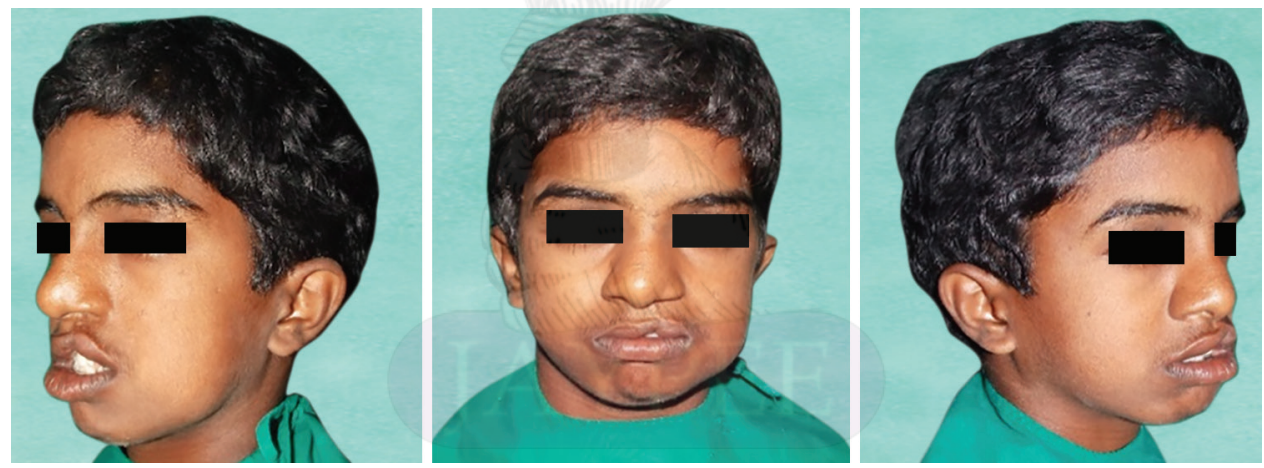

Fig. 1: Extraoral swelling and surgical scar mark of cleft lip surgery
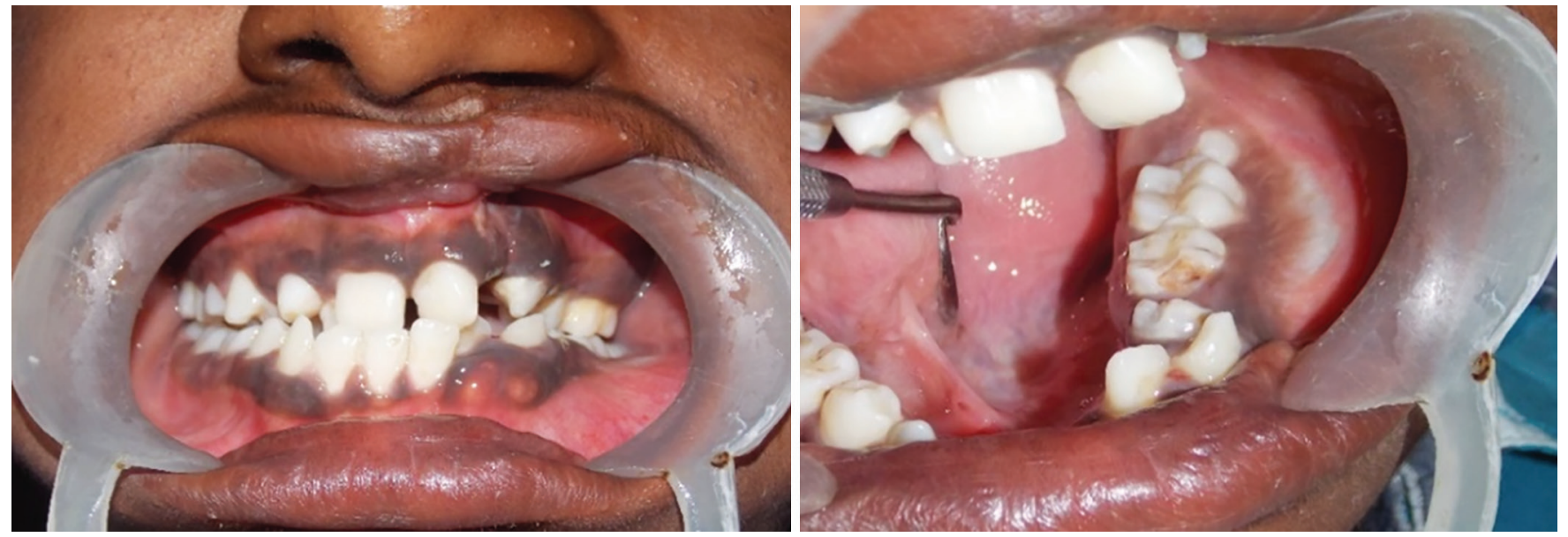

Fig. 2: Intraoral swelling and surgical scar mark of cleft alveolus surgery and multiple retained deciduous teeth 


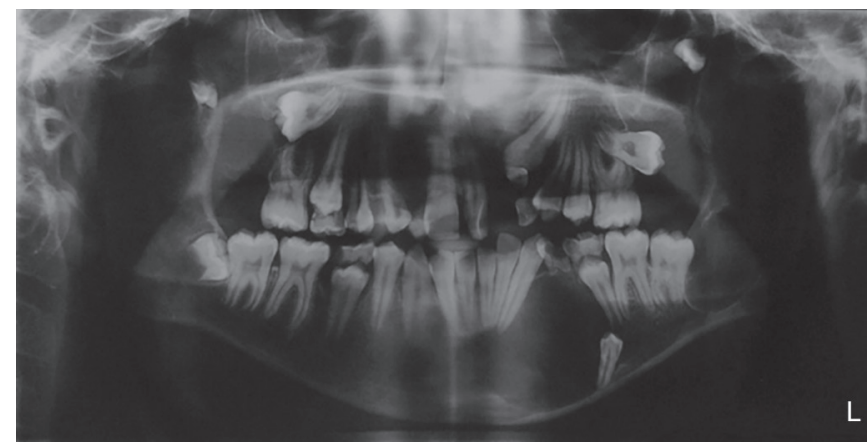

Fig. 3: OPG revealing multiple radiolucencies

both buccally and palatally was evident. Lesion occupies the entire maxillary sinus on both the right and the left side of the maxilla, with an evidence of bilocularity on the right side as lesion moves superiorly, along with impacted 17 and 28 (Figs 4 to 6).

The patient was subjected to incisional biopsy of all the cystic sites of the maxilla and mandible under general anesthesia. Biopsy results were suggestive of OKCs in relation to the anterior and the posterior mandible (Fig. 7) and the posterior right side of the maxilla (Fig. 8), while the left side of maxillary swelling associated with unerupted tooth was suggestive of hyperplastic dental follicle (Fig. 9).

Hematological investigations were within normal limits. Under general anesthesia, a staged surgical management was planned. In the first stage, the mandible was addressed by enucleation of cystic lesions along with the extraction of 74 and 75 and removal of unerupted 34 and 35 followed by three cycles of Carnoy's solution for a duration of 3 minutes per cycle. In the second stage, the maxilla was addressed and enucleation of cystic lesions was done followed by chemical cauterization using three cycles of Carnoy's solution.

All the lesions were sent for histopathological examination. The patient had an uneventful postoperative course. The patient has been kept on a regular follow-up since 2 years and the patient is asymptomatic till date.

$\mathrm{H}$-and E-stained sections of three cystic lesions, left mandible, both anterior and posterior regions, and right maxilla, showed the features of parakeratinized OKC with underlying connective tissue showing mild to moderate inflammatory infiltrate (Figs 7 and 8 ). $\mathrm{H}$ and $\mathrm{E}$ sections of the left maxilla revealed a few islands of odontogenic epithelium in dense collagenous stroma and one bit showing stromal components of immature cells, rich myxoid tissue with embryonic appearance, and inflamed cystic wall with no cystic lining concluding it to be hyperplastic dental follicle (Fig. 9). The patient had an uneventful postoperative course and has been kept on a regular follow-up since 2 years. No evidence of recurrence has been reported till date.

\section{Discussion}

OKC is a developmental cyst of odontogenic origin affecting oral and maxillofacial regions with a peak incidence in the second and the third decade of life. OKCs account for $11.2 \%$ of all the
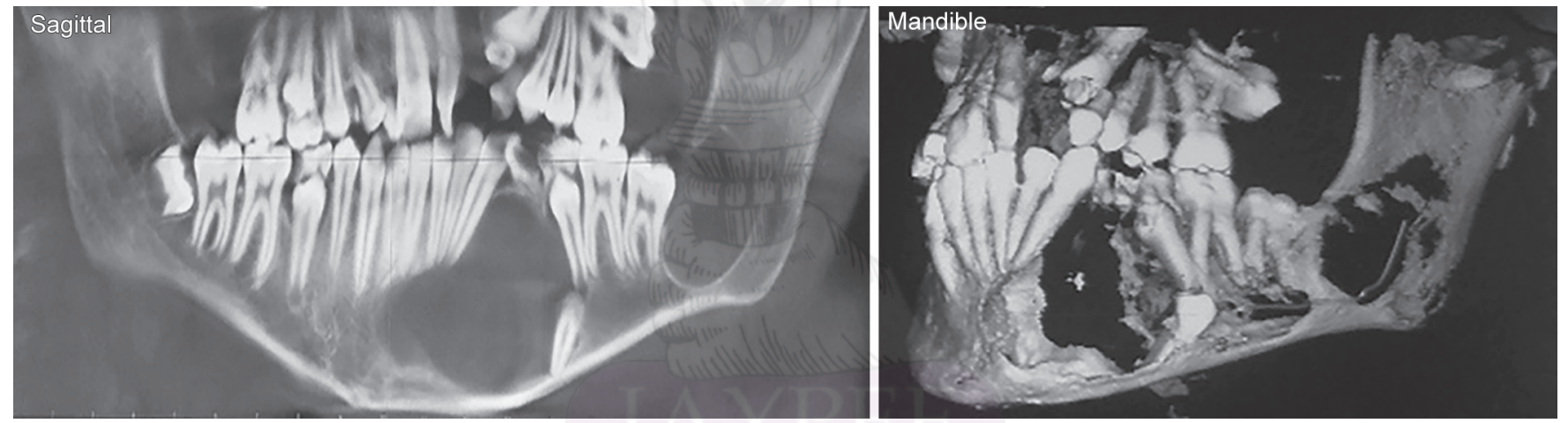

Fig. 4: CBCT scans revealing hypodense areas and multiple impacted teeth
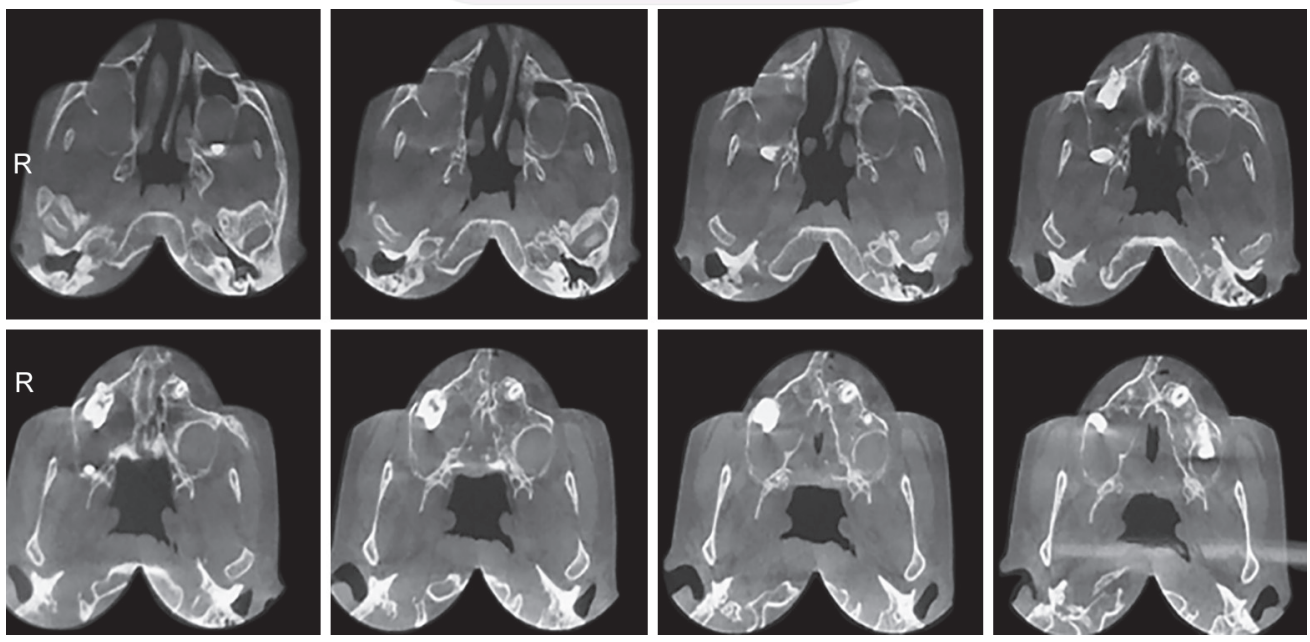

Fig. 5: CBCT scans of maxilla: right side, axial sections on the right side, reveals horizontally impacted 17 facing posteriorly, hypodense area of approximately $3 \mathrm{~cm}$ associated with an expansion of buccal as well as palatal cortical plates and lesion occupies the entire maxillary sinus, and bilocularity of lesion as it moves superiorly 

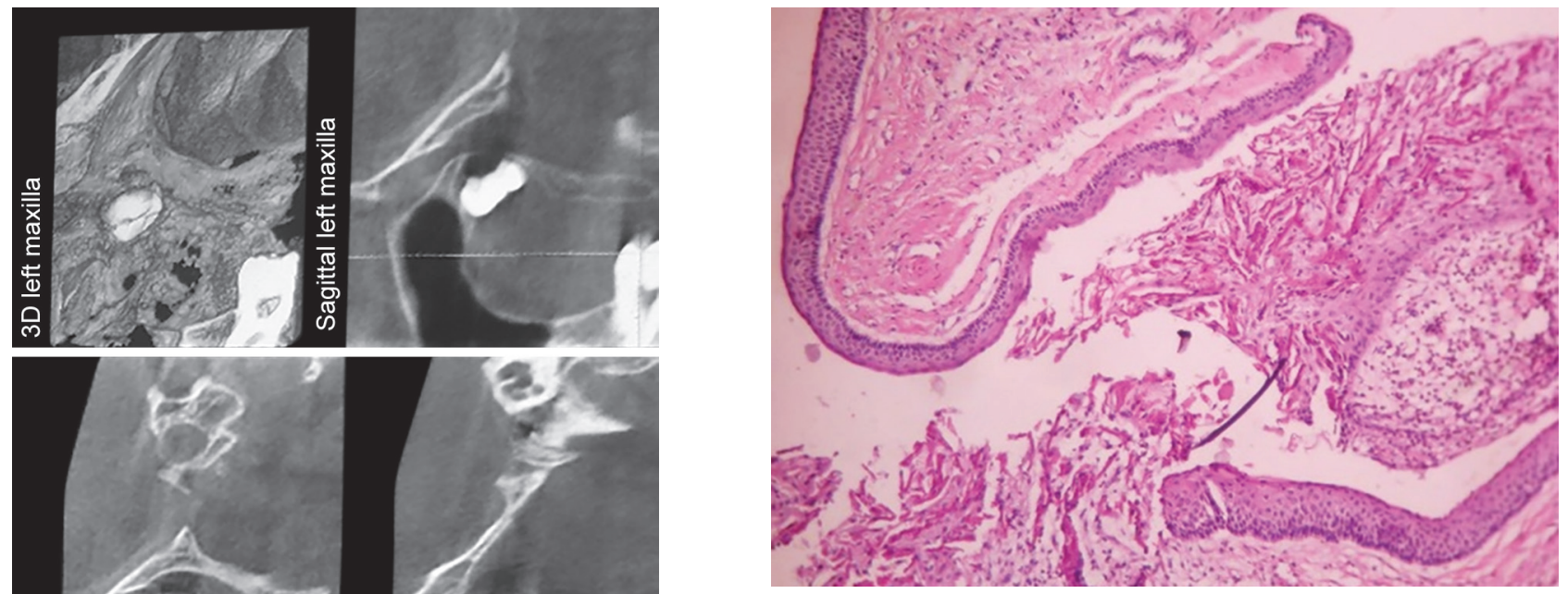

Fig. 7: Anterior and posterior mandible: $\mathrm{H}$ and $\mathrm{E}$ sections showing parakeratinized squamous epithelium with the prominent basal layer and with areas of desquamation

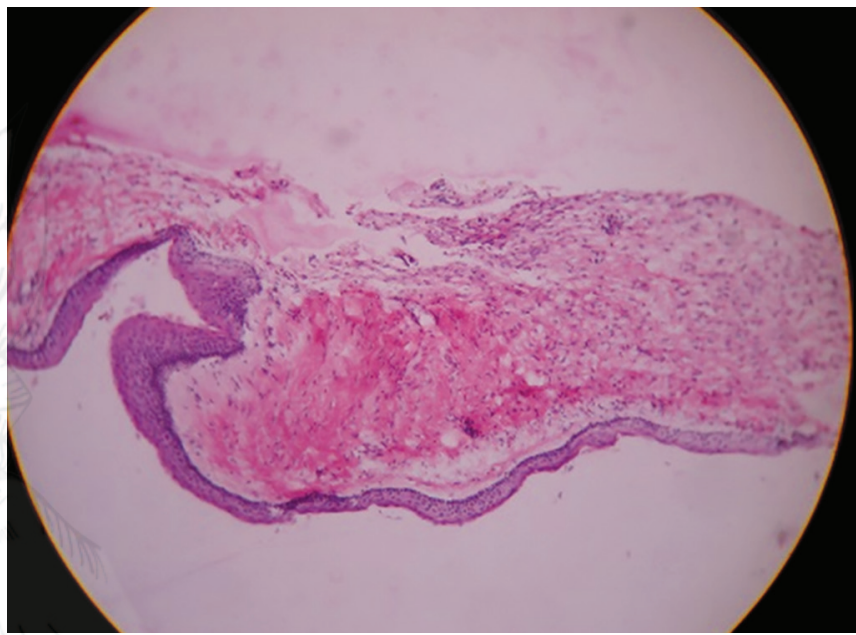

Fig. 8: Right maxilla: $H$ and $E$ sections showing parakeratinized squamous epithelium with areas of desquamation, elongation of rete ridges, and dense inflammation in the wall

Toller in 1967. In 1984, Ahlfors proposed that if OKCs were to be recognized and seen as a true, benign cystic epithelial neoplasm. This proposal raised a question mark regarding the treatment plan, whether to go for conservative management considering it to be cystic lesion or radical treatment considering it to be a benign tumor. In 2005, due to its aggressiveness, infiltrative behavior, histology, and genetic behavior, it was reclassified as KCOT by WHO. But recently, OKCs were again included in the odontogenic cyst category in the 4th edition of the WHO 2017 classification of odontogenic and maxillofacial bone tumors due to lack of evidences of it neoplastic nature as it was concluded that most cases of KCOT behave clinically as nonneoplastic lesions and are treated as cysts. Therefore, there was a consensus that they should be reclassified as OKC until there is more definite evidence for reclassifying them as KCOT (WHO Blue Book 2017). ${ }^{7}$

OKC can occur in sporadic or multiple forms. Brannon et al. reported that out of 312 cases of OKCs examined, $5.1 \%$ of the cases were associated with NBCCS and $5.8 \%$ were accompanied by multiple keratocysts, but without any other features of the 

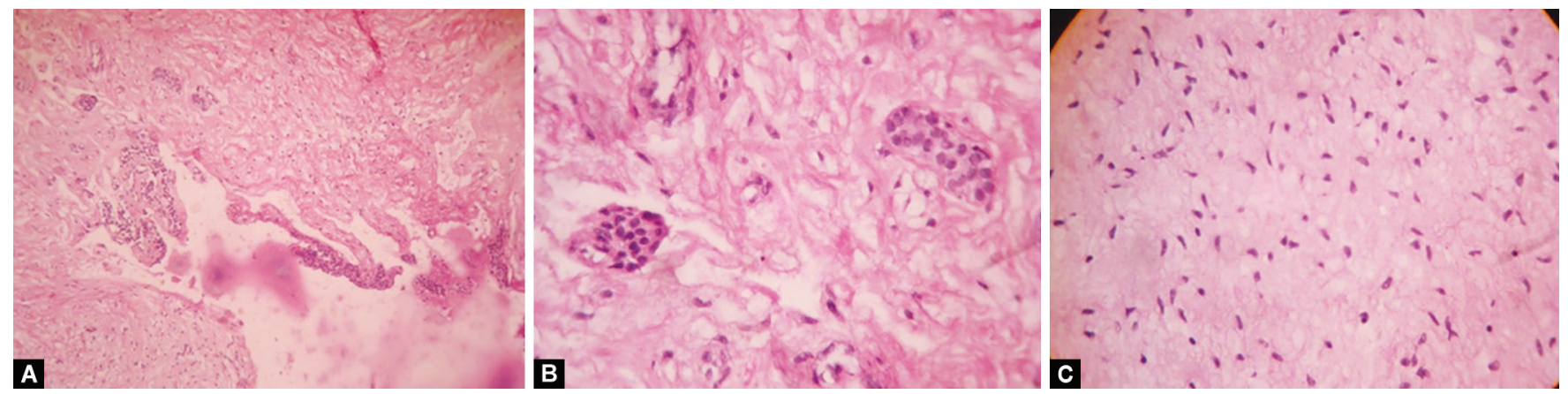

Figs $9 \mathrm{~A}$ to $\mathrm{C}:(\mathrm{A}-\mathrm{C})$ Left maxilla (hyperplastic dental follicle) $\mathrm{H}$ and $\mathrm{E}$ sections showing few islands of odontogenic epithelium in dense collagenous stroma ( $A$ and $B$ ) and one bit showing stromal components of immature cells in rich myxoid tissue with embryonic appearance (C)

syndrome. ${ }^{8}$ Our patient presented with multiple OKCs and with $\mathrm{h} / \mathrm{o}$ surgery for the cleft lip and cleft palate which is one of the minor criteria given by Kimons et al. to be considered as NBCCS. Other than this, the patient was fit and had no other features suggestive of syndrome.

Diagnostic criteria were given by Kimons et al. and Evans et al. ${ }^{4}$

\section{Major Criteria}

- More than two basal cell carcinomas or one in a patient $<20$ years of age

- Three or more plantar pits

- Bilamaller calcification of falxcerebri

- Odontokeratocyst of jaws proven histopathologically

- A first degree relative with NBCCS

- Bifid and fused ribs

Evans et al. added medulloblastoma and lymphomesentric cyst in major criteria.

\section{Minor Criteria}

- Macrocephaly

- Congenital malformations, hypertelorism, cleft lip/palate, and frontal bossing

- Skeletal deformities, Sprengel deformity, marked pectus deformity, and syndactyly of digits

- Radiological abnormalities, bridging of sella turcica, vertebral anomalies, and modeling defects of hands and feet

- Ovarian fibroma or medulloblastoma

NBCCS is an autosomal dominant syndrome with a high degree of penetrance and variable expressivity. About $65-75 \%$ of NBCCS patients have multiple OKCs that may be bilateral involving both the jaws and the maxillary and the mandibular canine region. ${ }^{8}$

Molecular basis of behavior associated with OKC is attributed to the underlying genetic event. NBCCS is associated with a mutation in the PTCH gene $9 q(22.3-q 31)$ that occurs in sporadic OKCs, as well as those associated with NBCCS. It has been suggested that a "two-hit" mechanism may underlie the variable expression of NBCCS and sporadic OKCs. In NBCCS, the basal cell carcinomas and keratocysts may arise as a consequence of a "first-hit" of allelic loss of PTCH gene within the precursor cell. ${ }^{3}$ The development of basal cell carcinoma and keratocysts in the absence of NBCCS reflects "two somatic hits" in which there are mutations of PTCH gene within locally susceptible cells that ultimately result in allelic loss. The absence of all the manifestations of NBCCS may be due to variability of the PTCH gene expression. ${ }^{9}$ Familial occurrence of syndromic multiple OKCs has also been reported in literature that emphasizes on taking familial history. ${ }^{9}$

Shear suggested that two cysts from one syndrome patient that occurred on opposite sides of the mandible had the same pattern of allelic loss, which suggests genetic mutation occurring at a very early stage of embryogenesis. ${ }^{9}$ Shear also proposed that that Knutson's "two-hit hypothesis of cancer development" may also explain the transformation of OKC into neoplasia., 3,4,8

The cyst spreads along the bone marrow resulting in more destruction before the appearance of any significant clinical manifestations. ${ }^{4,8}$ OKCs are locally aggressive, destructive, and highly recurrent with a recurrence rate of $3-60 \%$ that varies depending on the surgical approach taken. ${ }^{9}$ Literature reports a comparatively higher recurrence rates of OKC as a component of NBCCS due to the deep staining in the palisading basal layer that indicates a greater potential for proliferation, more common parakeratinization and satellite cysts, higher number of satellite rests of tumor, inhibition of apoptosis, angiogenesis, more solid areas of epithelial proliferation, and more odontogenic epithelial rests within the fibrous capsule. ${ }^{9}$

$\mathrm{Li}$ et al. reported the high levels of epidermal growth factor receptor (EGFR) expression in OKCs which supported the view that they have an intrinsic growth potential not present in other odontogenic cysts, also expression levels of TGF-a, EGF, and EGFR suggest the involvement of growth factors in their pathogenesis, and the correlation between $\mathrm{Ki}-67$ and PCNA reflects cell proliferation which has been considered to be histological markers and prognostic indicators. Expression of histological markers PCNA, ki67, and p53 is seen associated with the NBCCS syndrome. Partial expression of the PTCH gene may result in the occurrence of only multiple recurring OKCs without any associated systemic findings. ${ }^{10}$

Kuroyanagi et al. suggested ki-67 and argyrophilic nucleolar organizing regions (AgNORs) as marked prognostic indicators. Ki-67-labeling index consideration can be helpful in adjunctive surgical procedures. Also, overexpression of PCNA and along with $\mathrm{Ki}-67$ proves its highly aggressive behavior and recurrent nature. ${ }^{3,10}$

Histopathologically, OKCs reveal thin, friable wall, which makes it difficult to enucleate from the bone in one piece, and have small satellite cysts within the fibrous wall of cyst that favors the tendency to recur after treatment (Brannon, 1977; Gang et al., 2006). Radiographically, KCOT demonstrates a well-defined unilocular or multilocular radiolucency with smooth and often corticated margins. About $25-40 \%$ of cases are usually associated with an unerupted tooth involved in the lesion. ${ }^{2}$

Treatment of OKCs remains controversial till date. Morgan et al. categorized treatment modalities that are generally conservative or 
aggressive. Conservative approaches are cyst oriented and involves simple curettage, marsupialization, or enucleation with or without curettage. It has an advantage of preservation of anatomical structures (including teeth), which is advocated because OKCs commonly present in younger patients and it is applicable not only to all age groups but also to patients with NBCCS. Aggressive modalities involve peripheral ostectomy, chemical curettage with Carnoy's solution, cryotherapy or electrocautery, and block resection with or without preservation of continuity of jaw, thus, addressing the "neoplastic nature" of the OKCs and, generally, recommended for NBCCS cases, large OKCs, and recurrent lesion. ${ }^{5}$ Recent literature has reported that the suppression of sonic hedgehog $(\mathrm{SHH})$ signaling pathway might be effective for the treatment of OKCs. ${ }^{10,14}$ Choice of treatment varies depending on multiple factors: age, size, location of lesion, soft tissue involvement with a history of previous treatment, and histological variant. The ultimate goal is to choose the best modality that carries the least risk of recurrence and the least amount of morbidity. ${ }^{11}$ The conservative treatment can be chosen only if there is no coronoid invasion, no interruptive cortical lysis, and no tissue invasion., ${ }^{9,10}$

Stoelinga and Voorsmit etal advocated excision of the overlying mucosa followed by the application of the Carnoy's solution. 2,18-20 Carnoy's solution contains ferric chloride, absolute alcohol, chloroform, and glacial acetic acid that penetrates to a predictable time-dependent depth and, at the same time, the neurovascular bundle is protected with a coat of Vaseline. The application is done for 5 minutes which results in a bone penetration of $1.54 \mathrm{~mm}$ and a nerve penetration up to a depth of $0.15 \mathrm{~mm}$ and a mucosal depth of $5 \mathrm{~mm}$. In modified Carnoy's solution, chloroform has been omitted. Stoelinga advocated that the 3-minute application time to be safer. ${ }^{12}$

Cryosurgery involves the use of liquid nitrogen that has a unique ability to devitalize the bone in situ while leaving the inorganic framework intact. Both these techniques are advocated in killing any epithelial remnants and dental lamina within osseous keratocysts, thus, helping in reducing the rate of recurrences. Excision of overlying mucosa when combined with enucleation followed by chemical cauterization using Carnoy's solution or liquid nitrogen obtains the lowest recurrence rate, specifically for retromandibular trigone, posterior maxillary, and cortically perforated lesions. ${ }^{12,13}$

According to literature simple enucleation was reported to have a recurrence rate of $17-56 \%$, while simple enucleation combined with adjunctive therapy, such as the application of Carnoy's solution, decompression before enucleation, was reported to have a recurrence rate of $1-8.7 \%{ }^{16}$

Golgire et al. reported a case of 20 years old female patient having multiple OKCs in both the jaws simultaneously without any syndromic manifestation and also compared the syndromic and nonsyndromic cases in terms of clinical aspects, histopathological features, genetics, and recurrences. It was observed that syndromic cases are comparatively more aggressive that can probably be attributed to increased number of mitotic figures and satellite cysts with more expression of the PTCH gene. A recurrence rate of $63 \%$ was reported in syndromic cases, while $37 \%$ in the nonsyndromic cases. Also female predilection for syndromic cases as reported in literature and has been emphasized on pelvic ultrasonography in suspected females. ${ }^{17}$

Based on the available literature, it is clear that multiple OKCs may be the first and the only manifestation of NBCCS or any other syndrome that can appear a decade earlier before other symptoms appear. Since most of such cases appear during the second decade of life, the possibility of other symptoms of NBCCS or any other syndrome appearing in future cannot be excluded. So thorough clinical evaluation, appropriate investigations, formulation of a definitive treatment plan for that particular case and a strict posttreatment follow-up should be a line of overall management, as NBCCS is known for variability of expression and recurrences.

The recurrence rate up to $82 \%$ has been reported in syndromic cases and up to $61 \%$ in the nonsyndromic cases. ${ }^{4,8}$ However, the recurrence rate of $63 \%$ in syndromic and $37 \%$ in nonsyndromic cases have been reported in literature. ${ }^{17}$ So any patient presenting with OKCs should be further investigated for multiple OKCs and the one with multiple OKCs must be evaluated for features of NBCCS because the presence of multiple OKCs, at the same time, is not imperative rather it implies to the lifetime history of multiplicity and recurrences of lesions. ${ }^{4,9}$

Recognition of lesion/disease at an early stage provides a chance to evaluate the patient as well as asymptomatic siblings or relatives who can be subjected to genetic counseling and serial screening for the development of malignancies or any other symptoms of NBCCS.

\section{Conclusion}

Although our case does not fulfill the major and minor criteria's of syndrome, but considering the reported literature and clinical presentation, early age, multiple OKCs, involvement of both the jaws especially in posterior region, operated cleft lip and cleft palate, and histological correlation, we suggest our case to be partial expression of NBCCS. Our case is an addition to the previous number of such cases, highlighting the importance of thorough evaluation, diagnosis, treatment strategy, and strict follow-up of such cases so as to provide a better quality of life with a minimal amount of morbidity.

\section{Clinical Significance}

In any patient who is presenting with OKCs, there should be further investigation to rule out multiple OKCs and when presented with multiple OKCs, we should thoroughly investigate locally and systemically for any other major or minor criteria of NBCCS or signs of any other syndrome because syndromic cases are comparatively aggressive, infiltrative, and recurrent. Such patient has to be kept on a strict long-term follow-up because OKCs may be only or the first sign of a syndrome. Recurrences have been reported even after 10 years. A definite treatment protocol should be formulated depending on patient's age, number and size of lesion, remaining subsequent growth, and proximity to adjacent vital structures so as to provide a better quality of life with a minimal amount of morbidity.

\section{ACKNOWLedgment}

The authors thank Bapuji Dental College and Hospital Davangere, Department of General Pathology, JJMC Medical College, and Hospital Davangere.

\section{References}

1. Pogrel MA. The Keratocystic Odontogenic Tumor. Oral Maxillofac Surg Clin N Am 2013;25:21-30. DOI: 10.1016/j.coms.2012.11.003.

2. Madras J, Lapointe H. Keratocystic Odontogenic Tumour: Reclassification of the Odontogenic Keratocyst from Cyst to Tumour. J Can Dent Assoc 2008;74(2):165-165. 
3. Stoelinga PJW. Long-term follow-up on keratocysts treated according to a defined protocollnt. J Oral Maxillofac Surg 2001;30:14-25. DOI: 10.1054/ijom.2000.0027.

4. Rai S, Rana AS, et al. Multiple keratocystic odontogenic tumors in a non-syndromic minor patient: report of an unusual case. J Orofac Sci 2013;5(1):61-66. DOI: 10.4103/0975-8844.113708.

5. Guruprasad Y, Chauhan DS. Multiple odontogenic keratocysts in a nonsyndromic patient. J CranioMax Dis 2012;1(1):36-40. DOI: 10.4103/2278-9588.102500.

6. Tandon S, Khushboo P, et al. Pathogenesis of keratocystic odontogenic tumor-a review. TMU J Dent 2014;1(3):100-105.

7. Passi $D$, Singhal $D$, et al. Journey of okc from cyst to tumor to cyst again: comprehensive review who classification. Int J Curr Res 2017 July (07);9(7):54080-54086.

8. Ashoka CA. Non syndromic multiple keratocystic odontogenic tumour occurring in both the jaws: case report and review of literature. IOSR J Dent Med Sci 2015;14(1):35-41.

9. Kalia V, Nitin K, et al. The Syndromic multiple odontogenic keratocyst in siblings. A familial study. Ann Maxillofac Surg 2011;1(1):77-82. DOI: 10.4103/2231-0746.83156.

10. Selvi F, Tekkesin MS, et al. Keratocystic odontogenic tumors: predictive factors of recurrence by $\mathrm{Ki}-67$ and AgNOR labelling. Int J Med Sci 2012;9(4):262-268. DOI: 10.7150/ijms.4243.

11. Abdullha WA. Surgical treatment of keratocystic odontogenic tumour: a review article. Saudi Dent J 2011(23):61-65. DOI: 10.1016/ j.sdentj.2011.01.002.
12. Dixit S, Acharya $S$, et al. Multiple odontogenic keratocyst associated with gorlingoltz syndrome. Kathmandu Univ Med J 2009;7:414-418. DOI: 10.3126/kumj.v7i4.2765.

13. Moraissi AL, Pogrel MA, et al. Does the excision of overlying oral mucosa reduce the recurrence rate in the treatment of the keratocystic odontogenic tumor? A systematic review and meta-analysis. J Oral Maxillofac Surg 2016;74:1974-1982. DOI: 10.1016/j.joms.2016.03.043.

14. McGrath CJ, Myall RW. Conservative management of recurrent keratocysts in Basal-cell naevus syndrome. Aust Dent J 1997;42:399403. DOI: 10.1111/j.1834-7819.1997.tb06085.x.

15. Kurdekar RS, Prakash J, et al. Non syndromic odontogenic keratocysts: a rare case report. Natl J Maxillofac Surg 2013;4(1):90-93. DOI: 10.4103/0975-5950.117874.

16. Warburton G, Shihabi A, et al. Keratocystic odontogenic tumor (KCOT/OKC) - clinical guidelines for resection. J Maxillofac Oral Surg 2015;14(3):558-564. DOI: 10.1007/s12663-014-0732-7.

17. Golgire SM, Varekar AA. Non-Syndromic Multiple Odontogenic Keratocysts: A Case Report and Comparison with Syndromic Multiple Odontogenic Keratocysts. J Clin Case Rep 2016;6(6):818. DOI: 10.4172/2165-7920.1000818.

18. Voorsmit RACA, Stoelinga PJW, et al. The management of keratocysts. J Maxillofac Surg 1981;9:228-236.

19. Voorsmit RACA. The incredible keratocyst: A retrospective and perspective study [thesis]. Nijmegen: University of Nijmegen;1984.

20. Voorsmit RACA. The incredible Keratocyst: A new approach to treatment. Dtsch Zahnarztl Z 1985;40:641-644. 